\title{
COVID-19 e seus impactos aos profissionais de saúde atuantes na pandemia: Um estudo teórico reflexivo
}

COVID-19 and its impacts on health professionals active in the pandemic: A reflective theoretical study

COVID-19 y sus impactos en los profesionales de la salud activos en la pandemia: Un estudio teórico reflexivo

Recebido: 14/06/2021 | Revisado: 22/06/2021 | Aceito: 23/06/2021 | Publicado: 10/07/2021

Cláudio Manoel da Silva Barreto ORCID: https://orcid.org/0000-0001-9096-6893 Centro Universitário Estácio de Sergipe, Brasil E-mail: cmbarreto2017@outlook.com

Milena dos Anjos Castro Lessa ORCID: https://orcid.org/0000-0003-3428-1624 Centro Universitário Estácio de Sergipe, Brasil E-mail: myleenaacastro@gmail.com

Tainá da Costa Nunes Santos ORCID: https://orcid.org/0000-0001-8043-9619 Centro Universitário Estácio de Sergipe, Brasil E-mail: tainacosta1496@gmail.com

Ana Fátima Souza Melo de Andrade ORCID: https://orcid.org/0000-0002-7024-6175 Centro Universitário Estácio de Sergipe, Brasil E-mail: anafatimamelo@ hotmail.com

Felipe Tavares de Andrade ORCID: https://orcid.org/0000-0002-6395-2565 Universidade Federal de Sergipe, Brasil

E-mail: felipe.tavares.fisica@gmail.com

Weber de Santana Teles

ORCID: https://orcid.org/0000-0003-1770-8278

Centro de Hemoterapia de Sergipe, Brasil E-mail: arteecura@hotmail.com Max Cruz da Silva

ORCID: https://orcid.org/0000-0002-6944-5986 Faculdade Pio Décimo, Brasil E-mail: maxlfi@hotmail.com

Ruth Cristini Torres

ORCID: https://orcid.org/0000-0002-8664-192X Instituto de hematologia e hemoterapia de Sergipe, Brasil E-mail: ruthcristini@gmail.com

Angela Maria Melo Sá Barros

ORCID: https://orcid.org/0000-0003-4087-3247 Universidade Federal do Rio de Janeiro, Brasil

E-mail: angelsamelo@hotmail.com

Marcel Vinícius Cunha Azevedo ORCID: https://orcid.org/0000-0002-5312-3333 Centro Universitário Estácio de Sergipe, Brasil

E-mail: marcelvinicius49@gmail.com

Rute Nascimento da Silva ORCID: https://orcid.org/0000-0002-2719-1623 Universidade Tiradentes, Brasil E-mail: silva_rute@hotmail.com

Paulo Celso Curvelo Santos Junior ORCID: https://orcid.org/0000-0001-5834-6782 Universidade Tiradentes, Brasil E-mail: paulo.curvelo.jr@gmail.com

Taíssa Alice Soledade Calasans ORCID: https://orcid.org/0000-0003-0460-4437 Universidade Tiradentes, Brasil E-mail: taissa.asc@gmail.com 


\begin{abstract}
Resumo
Diante do vírus da COVID-19 se fizeram necessárias várias medidas preventivas para evitar a contaminação pela doença. A OMS preconiza o uso de EPIs, a higienização das mãos e o isolamento social, visando à minimização do contágio. Para os profissionais de saúde não existe a possibilidade de isolamento, visto que estão na linha de frente contra a COVID-19. Objetivou-se identificar os danos causados nos profissionais de saúde atuantes na linha de frente contra a Pandemia da COVID-19. Trata-se de um estudo teórico reflexivo com abordagem qualitativa, construído com base na leitura crítica de referências nacionais e internacionais, existentes em publicações, acerca dos danos causados nos profissionais de saúde, durante a pandemia da COVID-19. Foram utilizadas as seguintes bases de dados: BVS e PubMed. Foram selecionados 17 estudos, distribuídos em âmbito nacional $(76,47 \%)$ e internacional $(23,53 \%)$. Os resultados obtidos; evidenciaram a enfermagem enquanto categoria profissional que sofreu maior impacto, seguida pela medicina; houve predominância de acometimento em profissionais do sexo feminino, entre 40 - 50 anos, de raça/cor branca; os principais sintomas relacionados à sua jornada de trabalho e exposição ocupacional foram: medo, insegurança, incertezas, desesperanças, ansiedade e depressão. Portanto, a assistência exige a implementação de estratégias voltadas para os profissionais de saúde atuantes na linha de frente.
\end{abstract}

Palavras-chave: Coronavírus; Pandemias; Profissionais de saúde; Cuidados de enfermagem; Impactos na saúde.

\begin{abstract}
In view of the COVID-19 virus, several preventive measures were necessary to avoid the contamination by the disease. WHO recommends the use of PPE, hand hygiene and social isolation, aiming to minimize contagion. For health professionals there is no possibility of isolation, as they are on the front line against COVID-19. The objective was to identify the damage caused to health professionals working on the front line against the COVID-19 Pandemic. This is a reflective theoretical study with a qualitative approach, built on the basis of critical reading of national and international references, existing in publications, about the damage caused to health professionals during the COVID19 pandemic. The following databases were used: BVS and PubMed. 17 studies were selected, distributed nationally (76.47\%) and internationally $(23.53 \%)$. The results obtained evidenced nursing as a professional category that suffered the greatest impact, followed by medicine; there was a predominance of involvement in female professionals, aged between 40 - 50 years, of white race/color; the main symptoms related to their working hours and occupational exposure were: fear, insecurity, uncertainty, hopelessness, anxiety and depression. Therefore, assistance requires the implementation of strategies aimed at health professionals working on the front lines.
\end{abstract}

Keywords: Coronavirus; Pandemics; Health professionals; Nursing care; Health impacts.

\title{
Resumen
}

Ante el virus COVID-19, fueron necesarias varias medidas preventivas para evitar la contaminación por la enfermedad. La OMS recomienda el uso de EPP, higiene de manos y aislamiento social, con el objetivo de minimizar el contagio. Para los profesionales de la salud no hay posibilidad de aislamiento, ya que están en primera línea contra el COVID-19. El objetivo fue identificar el daño causado a los profesionales de la salud que trabajan en primera línea contra la Pandemia COVID-19. Se trata de un estudio teórico reflexivo con enfoque cualitativo, construido a partir de una lectura crítica de referencias nacionales e internacionales, existentes en publicaciones, sobre el daño causado a los profesionales de la salud durante la pandemia de COVID-19. Se utilizaron las siguientes bases de datos: BVS y PubMed. Se seleccionaron 17 estudios, distribuidos a nivel nacional $(76,47 \%)$ e internacional $(23,53 \%)$. Los resultados obtenidos evidenciaron a la enfermería como categoría profesional de mayor impacto, seguida de la medicina; predominó la participación de mujeres profesionales, con edades comprendidas entre los 40 y los 50 años, de raza / color blanco; los principales síntomas relacionados con su jornada laboral y exposición ocupacional fueron: miedo, inseguridad, incertidumbre, desesperanza, ansiedad y depresión. Por tanto, la asistencia requiere la implementación de estrategias dirigidas a los profesionales de la salud que trabajan en primera línea.

Palabras clave: Coronavirus; Pandemias; Profesionales de la salud; Cuidado de enfermera; Impactos en la salud.

\section{Introdução}

No mês de dezembro de 2019, uma onda de doenças respiratórias acometeu uma província de Hubei, na China. Sendo identificado pelo Centro de Controle e Prevenção de Doenças (CDC) do país, o surto de um vírus pertencente à família Coronaviridae e denominado SARS-CoV-2 (2019-nCoV). Pelo seu alto potencial de transmissão e contágio por vias respiratórias, a Organização Mundial de Saúde (OMS) declarou a pandemia de COVID-19 no dia 11 de março de 2020 (WHO, 2020). Segundo dados da OMS (2021), até o dia 19 de abril de 2021 foram notificados 141.057.106 casos confirmados de COVID-19, 3.015.043 mortes e 792.796.083 doses de vacina adminstradas.

O Brasil recebeu a primeira notificação no dia 26 de fevereiro de 2020, oficializando o início do contágio da doença, através do retorno de um indivíduo da Itália para a sua cidade de residência São Paulo, testando positivo para COVID-19. Em 
março, o Governo do Estado de São Paulo, epicentro da pandemia no país, promulgou o Decreto $\mathrm{n}^{\circ}$ 64.881, no qual se definiram medidas de isolamento social e quarentena estadual (Araújo et al., 2021). Até o dia 19 de abril de 2021, foram confirmados 12.460 .712 casos e 374.682 óbitos (Brasil, 2021).

A patologia supracitada apresenta diversas sintomatologias destacando-se: febre, dispneia, tosse seca, cefaleia, diarreia, algias musculares, anosmia e ageusia. Não obstante, a elevada transmissibilidade da doença tem como um dos fatores o quantitativo de casos considerados assintomáticos, cerca de $80 \%$. Isso deve-se ao fato de que, independente da presença ou ausência de sintomas, os indivíduos infectados podem transmiti-la, tornando-a de alta contagiosidade (Yu et al., 2020).

Mesmo diante dos altos índices epidemiológicos, para os profissionais de saúde, não existe a possibilidade de isolamento, pois são estes atuantes na linha de frente contra a COVID-19, incorrendo em maior risco de contágio, dado pela escassez de equipamentos de proteção individual, desconforto e lesão ao utilizá-los, visto que seu uso prolongado não fazia parte do cotidiano. (Aydogdu, 2020).

Estudo realizado por Koh (2020) aponta a alta incidência de complicações cutâneas relacionadas às medidas de prevenção entre profissionais de saúde que tratam pacientes com infecção pela COVID-19, havendo, como consequência, uma redução do uso de EPIs devido à ulceração cutânea. Dos profissionais estudados, 97\% apresentavam lesões em ponte nasal, mãos, bochechas e testa. E devido à frequente higienização das mãos, uma incidência de dermatite na região tornou-se ascendente.

Anvian (2020) relata fatores que contribuiram para o sofrimento psicológico dos profissionais de saúde como: desgaste emocional, exaustão física, escassez de EPIs, ansiedade, depressão, cargas de trabalho excessivas e; acesso limitado a serviços de saúde mental. Diante disso, o estudo é de ampla relevância, uma vez que contribuirá para reflexão sobre os danos causados aos profissionais de saúde atuantes contra a Pandemia da COVID-19, possibilitando um olhar direcionado a esse profissional. Por outro viés, permitirá a elaboração e estruturação da atenção à saúde do trabalhador, com uma abordagem eficiente quanto aos determinantes de saúde relacionados à exposição ocupacional a agentes biológicos. Deste modo, o presente estudo objetivou-se identificar os danos causados aos profissionais de saúde atuantes na linha de frente contra a Pandemia da COVID-19.

\section{Metodologia}

Trata-se de um estudo integrativo da literatura, que tem como propósito agregar conteúdos científicos descritivos com abordagem qualitativa, estruturados a partir das plataformas científicas com base nas referências nacionais e internacionais, abordando os danos causados pela COVID-19 aos profissionais de saúde durante a pandemia. Tornando-se possível analisar as evidências que se encontram disponíveis nas plataformas científicas e contribuir com a ampliação do conhecimento quanto ao assunto.

Utilizaram-se como critérios de inclusão, artigos originais, nos idiomas português, inglês e espanhol, respeitando o limite de tempo da publicação entre os anos de 2020 e 2021 e que abordassem a temática referente aos impactos causados aos profissionais de saúde atuantes na linha de frente contra a pandemia da COVID-19.

Para a concepção do presente estudo, foram acompanhadas as sequências: identificação do tema, estabelecimento dos critérios de inclusão e exclusão dos artigos, definição de dados que seriam retirados, e a revisão. A pesquisa e a revisão foram realizadas no período entre agosto de 2020 e abril de 2021.

A escolha e discussão do tema norteou-se nas seguintes categorias metodológicas: O cenário da pandemia da COVID19 no Brasil e no mundo, a contaminação dos profissionais de saúde envolvidos diretamente nos cuidados dos pacientes sintomáticos e diagnosticados com a infecção provocada pela COVID-19, as graves e prejudicias consequências aos que atuam na assistência dos pacientes, estratégias para a promoção de saúde dos profissionais de saúde que atuam na linha de frente. 
A busca pelos artigos foi realizada por meio das bases de dados: Biblioteca Virtual em Saúde (BVS), Literatura Latino-Americana e do Caribe em Ciências da Saúde (LILACS), Medical Literature Analysis and Retrieval Sistem online (Medline), $e$ National Library of Medicine and National Institute of Health - USA (PubMed) e Scientific Electronic Library Online (SciELO).

Foram utilizados os seguintes descritores em saúde consultados na plataforma DeCS (Descritores em Ciências da Saúde): Coronavírus, pandemias, profissionais de saúde, cuidados de enfermagem, impactos na saúde.

Dos 31 estudos identificados, 17 foram selecionados, sendo 11 da base de dados Biblioteca Virtual em Saúde e seis da base de dados PubMed. Os 14 estudos excluídos não obedeceram aos critérios de inclusão propostos nesta pesquisa por não apresentarem relação com a metodologia proposta.

Os dados obtidos seguiram os princípios éticos, como também a Lei dos Direitos Autorais nº 12.853/13, que dispõem sobre a gestão coletiva de direitos da mesma natureza.

\section{Resultados}

A apresentação dos resultados obtidos; encontram-se exibidos de modo descritivo e organizados através de gráficos e quadros, o que possibilitam melhor interpretação dos dados. Foram selecionados 13 estudos distribuídos em âmbito nacional $(76,47 \%)$ e 4 quatro em âmbito internacional $(23,53 \%)$. Os estudos brasileiros; ocorreram nas regiões Sudeste, com $75 \%$ de artigos pesquisados, $75 \%$ e Sul, num percentual de 25\%, conforme a Figura 1.

Figura 1: Distribuição dos artigos com base na origem de publicação.
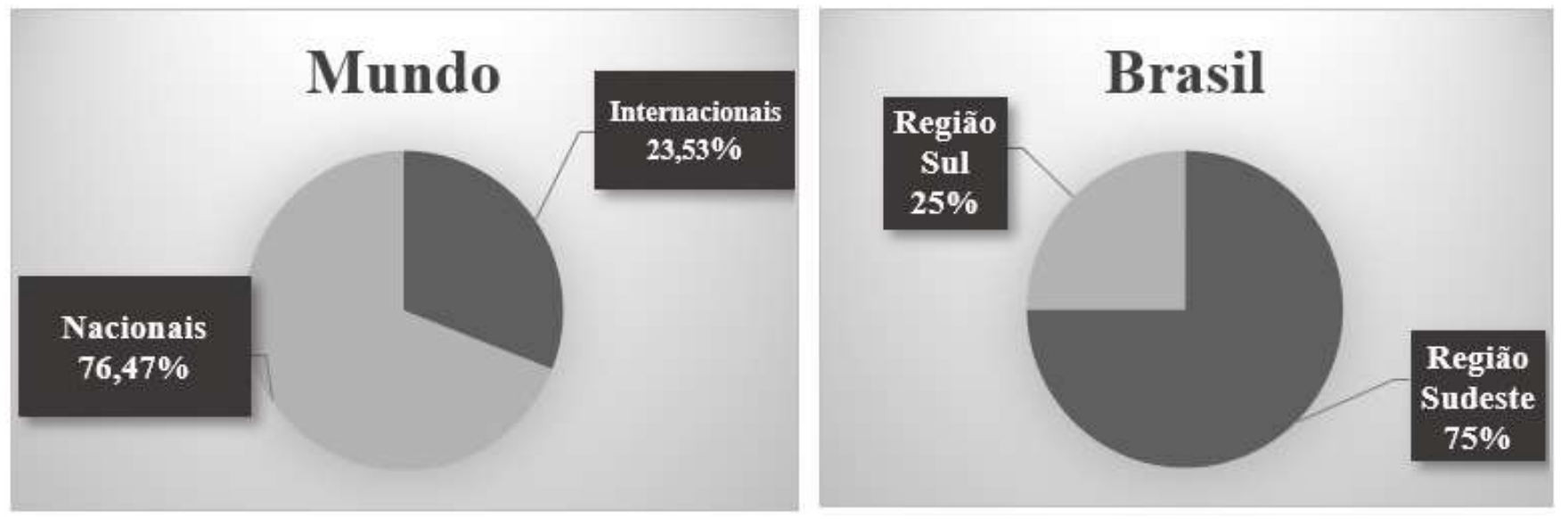

Fonte: Autores.

A fim de apresentar estatisticamente os resultados obtidos no referente estudo, elaborou-se um gráfico exibido de modo descritivo, destacando as respectivas bases de dados utilizadas. Dos 17 estudos selecionados, 64,7\% foram extraídos da Biblioteca Virtual em Saúde (BVS) e 35,3\% da National Library of Medicine and National Institute of Health - USA (PubMed), conforme demonstrado na Figura 2 
Figura 2: Distribuição dos estudos conforme as bases de dados utilizadas.

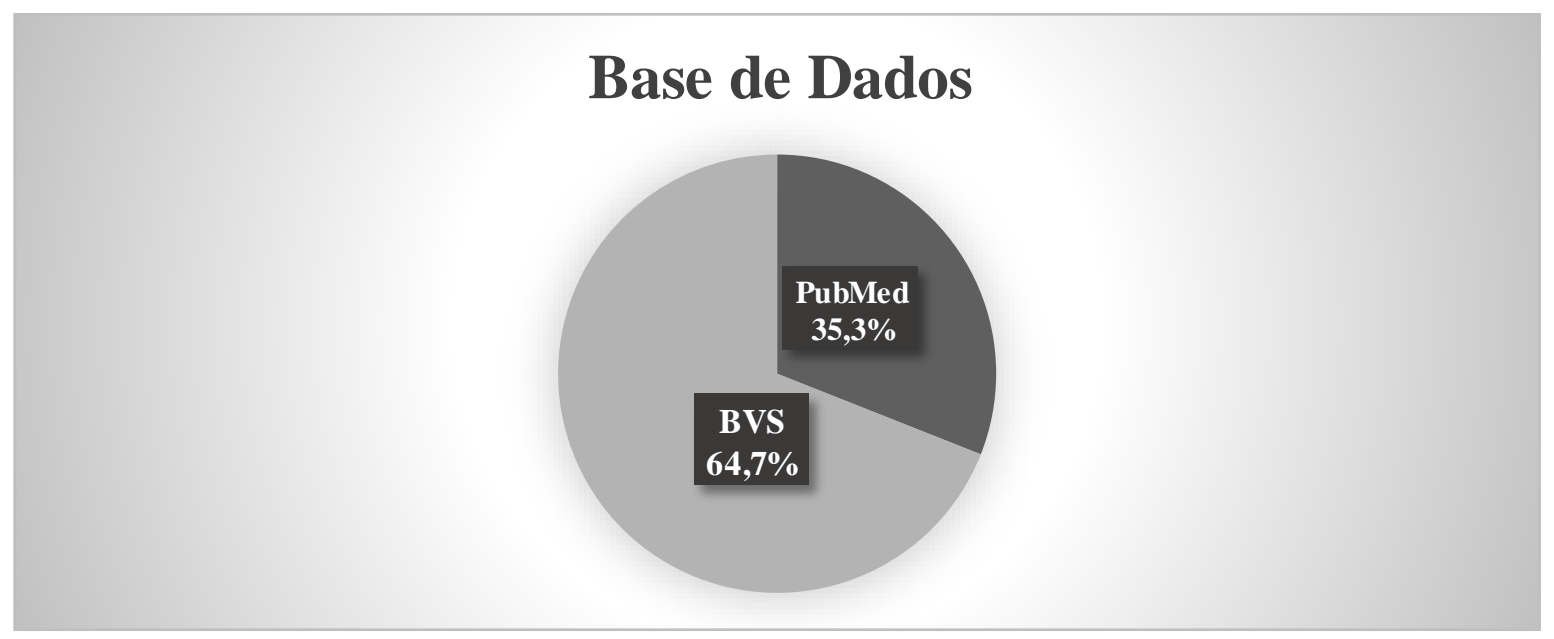

Fonte: Autores.

Para proferir melhor análise, foi elaborádo um quadro sinóptico com título, autores, metodologia e objetivos, o que possibilita a compreensão e síntese dos conteúdos abordados com relação ao tema proposto-,-conforme demonstrado no Quadro 1.

Quadro 1: Quadro sinóptico com a caracterização dos estudos selecionados.

\begin{tabular}{|c|c|c|c|}
\hline Nome do artigo & Autor/ ano & Método & Objetivo \\
\hline $\begin{array}{l}\text { Como o Brasil pode deter a } \\
\text { COVID- } 19 \text {. }\end{array}$ & $\begin{array}{l}\text { Oliveira et al. } \\
\qquad(2020)\end{array}$ & $\begin{array}{l}\text { Estudo transversal descritivo e } \\
\text { qualitativo. }\end{array}$ & $\begin{array}{l}\text { Apresentar as estratégias e ações adotadas } \\
\text { pelo Ministério da Saúde do Brasil para deter } \\
\text { a COVID-19. }\end{array}$ \\
\hline $\begin{array}{l}\text { A saúde dos profissionais de } \\
\text { saúde no enfrentamento da } \\
\text { pandemia de Covid- } 19 .\end{array}$ & $\begin{array}{l}\text { Teixeira et al. } \\
\text { (2020) }\end{array}$ & $\begin{array}{l}\text { Estudo elaborado a partir da } \\
\text { síntese de evidências científicas, } \\
\text { baseado em revisão de artigos } \\
\text { publicados em revistas nacionais e } \\
\text { internacionais }\end{array}$ & $\begin{array}{l}\text { Sistematizar um conjunto de evidências } \\
\text { científicas apresentadas em artigos } \\
\text { internacionais que identificam os principais } \\
\text { problemas que estão afetando os } \\
\text { profissionais de saúde envolvidos } \\
\text { diretamente no enfrentamento da pandemia } \\
\text { de COVID-19 e apontam ações e estratégias } \\
\text { para a proteção e a assistência à saúde desses } \\
\text { profissionais. }\end{array}$ \\
\hline $\begin{array}{l}\text { A saúde dos trabalhadores de } \\
\text { saúde no enfrentamento da } \\
\text { pandemia da Covid- } 19 \text {. }\end{array}$ & $\begin{array}{l}\text { Lima et al. } \\
\text { (2020) }\end{array}$ & $\begin{array}{l}\text { Estudo elaborado a partir de uma } \\
\text { síntese de evidências científicas, } \\
\text { baseado em revisão de artigos } \\
\text { publicados em revistas nacionais e } \\
\text { internacionais. }\end{array}$ & $\begin{array}{l}\text { Identificar os principais problemas de saúde } \\
\text { (e de saúde mental) correlacionados à } \\
\text { pandemia da Covid-19; as especificidades } \\
\text { das diferentes categorias profissionais e } \\
\text { marcadores sociais, como raça, gênero e } \\
\text { classe, dentre outros; as propostas, ações e } \\
\text { estratégias que vêm sendo adotadas para o } \\
\text { enfrentamento desses problemas, } \\
\text { particularmente as ações voltadas para a } \\
\text { promoção, proteção e assistência à saúde dos } \\
\text { trabalhadores de saúde que estão na linha de } \\
\text { frente do combate à pandemia. }\end{array}$ \\
\hline $\begin{array}{l}\text { COVID - 19: medidas de } \\
\text { prevenção de lesão por pressão } \\
\text { ocasionadas por equipamentos } \\
\text { de proteção individual em } \\
\text { profissionais da saúde. }\end{array}$ & $\begin{array}{l}\text { Luz et al. } \\
\text { (2020) }\end{array}$ & $\begin{array}{l}\text { Revisão integrativa, com estudos } \\
\text { publicados entre os anos de } 2000 \text { a } \\
2020 .\end{array}$ & $\begin{array}{l}\text { Identificar na literatura, as medidas } \\
\text { preventivas para desenvolver lesão por } \\
\text { pressão em profissionais de saúde. }\end{array}$ \\
\hline
\end{tabular}




\begin{tabular}{|c|c|c|c|}
\hline $\begin{array}{l}\text { The mental health of medical } \\
\text { workers in Wuhan, China } \\
\text { dealing with the } 2019 \text { novel } \\
\text { coronavirus. }\end{array}$ & $\begin{array}{l}\text { Kang et al. } \\
\text { (2020) }\end{array}$ & $\begin{array}{l}\text { Relatos de experiências dos abalos } \\
\text { mental ocorridos nos profissionais } \\
\text { médicos em Wuhan na China. }\end{array}$ & $\begin{array}{l}\text { Identificar os transtornos psíquicos dos } \\
\text { médicos frente a pandemia COVID-19. }\end{array}$ \\
\hline $\begin{array}{l}\text { Utilização de equipamentos de } \\
\text { proteção individual em época de } \\
\text { COVID-19. }\end{array}$ & $\begin{array}{l}\text { Carvalho et al. } \\
\qquad(2020)\end{array}$ & $\begin{array}{l}\text { Estudo de revisão, a partir do ano } \\
\text { de 2020, utilizando as bases de } \\
\text { dados SciELO, PubMed e } \\
\text { Medline. }\end{array}$ & $\begin{array}{l}\text { Atualizar os profissionais de saúde quanto } \\
\text { aos equipamentos de proteção individual } \\
\text { (EPIs), os tipos de equipamentos, o modo de } \\
\text { utilização, além de sua eficácia e eficiência. }\end{array}$ \\
\hline $\begin{array}{l}\text { Consensus of Chinese experts on } \\
\text { protection of skin and mucous } \\
\text { membrane barrier for healthcare } \\
\text { workers fighting against } \\
\text { coronavirus disease } 2019 \text {. }\end{array}$ & $\begin{array}{l}\text { Yan et al. } \\
\text { (2020) }\end{array}$ & $\begin{array}{l}\text { Estudo elaborado a partir de uma } \\
\text { síntese de evidências científicas, } \\
\text { para identificar medida de proteção } \\
\text { contra a COVID-13 }\end{array}$ & $\begin{array}{l}\text { Orientar os profissionais de saúdes na linha } \\
\text { de frente à pandemia quanto as medidas de } \\
\text { proteção. }\end{array}$ \\
\hline $\begin{array}{l}\text { Análise da saúde mental na } \\
\text { população geral durante a } \\
\text { pandemia de Covid-19. }\end{array}$ & $\begin{array}{l}\text { Ladeia et al. } \\
\quad(2020)\end{array}$ & $\begin{array}{l}\text { Revisão sistemática com buscas } \\
\text { nas plataformas Medline/PubMed. }\end{array}$ & $\begin{array}{l}\text { Analisar a influência da pandemia da } \\
\text { COVID-19 na saúde mental da população em } \\
\text { geral; e avaliar os fatores que levam e } \\
\text { agravam os transtornos psicológicos e fatores } \\
\text { que trazem tranquilidade à população. }\end{array}$ \\
\hline $\begin{array}{l}\text { COVID-19: por que a proteção } \\
\text { da saúde dos trabalhadores e } \\
\text { trabalhadoras da saúde é } \\
\text { prioritária no combate à } \\
\text { pandemia? }\end{array}$ & $\begin{array}{l}\text { Helioterio et al. } \\
\qquad(2020)\end{array}$ & $\begin{array}{l}\text { Estudo transversal descritivo e } \\
\text { qualitativo. }\end{array}$ & $\begin{array}{l}\text { Sumarizar e sistematizar aspectos relativos às } \\
\text { condições de trabalho e de saúde dos/as } \\
\text { trabalhadores/as da saúde nessa pandemia, } \\
\text { enfatizando a situação no Brasil, experiências } \\
\text { exitosas na proteção do trabalho em saúde } \\
\text { em outros países e recomendações para o } \\
\text { contexto brasileiro. }\end{array}$ \\
\hline $\begin{array}{l}\text { Estratégias de Prevenção frente à } \\
\text { Pandemia por Covid-19. }\end{array}$ & Ramalho (2020) & $\begin{array}{l}\text { Estudo realizado por meio de uma } \\
\text { revisão das evidências científicas, } \\
\text { extraídas de Consensos e } \\
\text { Diretrizes nacionais } \\
\text { internacionais, e e foram } \\
\text { selecionadas as principais medidas } \\
\text { para a proteção da pele, que } \\
\text { poderiam ser adotadas pelos } \\
\text { profissionais de saúde durante a } \\
\text { assistência a pacientes com } \\
\text { suspeita ou confirmação do } \\
\text { COVID-19. }\end{array}$ & $\begin{array}{l}\text { Divulgar este manual com as principais } \\
\text { recomendações para a prevenção de lesões } \\
\text { por pressão relacionadas a dispositivos } \\
\text { médicos (LPRDM) em profissionais de } \\
\text { saúde. }\end{array}$ \\
\hline $\begin{array}{l}\text { A pandemia de COVID-19 no } \\
\text { Brasil: crônica de uma crise } \\
\text { sanitária anunciada }\end{array}$ & $\begin{array}{l}\text { Wernek e } \\
\text { Carvalho } \\
(2020)\end{array}$ & $\begin{array}{l}\text { Estudo elaborado a partir da } \\
\text { síntese de evidências científicas, } \\
\text { baseado em revisão de artigos } \\
\text { publicados em revistas nacionais e } \\
\text { internacionais }\end{array}$ & $\begin{array}{l}\text { Identificar a crise sanitária causada pela } \\
\text { pandemia COVID-19 }\end{array}$ \\
\hline $\begin{array}{l}\text { Descrição dos } \quad \text { casos } \\
\text { hospitalizados pela COVID-19 } \\
\text { em profissionais de saúde nas } \\
\text { primeiras nove semanas da } \\
\text { pandemia, Brasil, } 2020\end{array}$ & $\begin{array}{l}\text { Duarte et al. } \\
\quad(2020)\end{array}$ & $\begin{array}{l}\text { Estudo descritivo de tipo série de } \\
\text { casos; foram incluídos aqueles } \\
\text { com adoecimento entre } 21 \text { de } \\
\text { fevereiro e } 15 \text { de abril de } 2020, \\
\text { registados no Sistema de } \\
\text { Informação de Vigilância da Gripe } \\
\text { (SIVEP-Gripe). }\end{array}$ & $\begin{array}{l}\text { Descrever os casos hospitalizados pela } \\
\text { COVID-19 em profissionais de saúde no } \\
\text { Brasil. }\end{array}$ \\
\hline $\begin{array}{lcr}\text { Cartilha sobre lesões de pele } \\
\text { relacionadas ao uso de } \\
\text { dispositivos } & \text { médicos no } \\
\text { enfrentamento ao COVID-19: } \\
\text { medidas de prevenção e } \\
\text { tratamento }\end{array}$ & $\begin{array}{l}\text { Dutra E Xavier } \\
\qquad(2020)\end{array}$ & $\begin{array}{l}\text { Estudo elaborado a partir de uma } \\
\text { síntese de evidências científicas, } \\
\text { baseado em revisão de artigos } \\
\text { publicados em revistas nacionais e } \\
\text { internacionais. }\end{array}$ & $\begin{array}{l}\text { Orientar os profissionais da saúde na linha de } \\
\text { frente durante a pandemia sobre o uso correto } \\
\text { dos EPIs }\end{array}$ \\
\hline
\end{tabular}




\begin{tabular}{|c|c|c|c|}
\hline $\begin{array}{l}\text { Saúde mental de profissionais de } \\
\text { enfermagem durante a pandemia } \\
\text { de COVID-19: recursos de } \\
\text { apoio. }\end{array}$ & $\begin{array}{l}\text { Toescher et al. } \\
\qquad(2020)\end{array}$ & $\begin{array}{l}\text { Estudo reflexivo, produzido com } \\
\text { base na formulação discursiva } \\
\text { acerca da saúde mental dos } \\
\text { profissionais de enfermagem frente } \\
\text { a pandemia de coronavírus, } \\
\text { correlacionado com as atuais } \\
\text { recomendações de suporte } \\
\text { psicológico do Ministério da } \\
\text { Saúde, Organização Mundial da } \\
\text { Saúde, Organização Pan- } \\
\text { Americana da Saúde e Fundação } \\
\text { Oswaldo Cruz. }\end{array}$ & $\begin{array}{l}\text { Refletir sobre as implicações da pandemia de } \\
\text { coronavírus na saúde mental dos } \\
\text { profissionais de enfermagem e os principais } \\
\text { recursos de apoio em desenvolvimento }\end{array}$ \\
\hline $\begin{array}{l}\text { A saúde mental da enfermagem } \\
\text { no enfrentamento da COVID-19 } \\
\text { em um hospital universitário } \\
\text { regional. Revista Brasileira de } \\
\text { Enfermagem. Brasília. } 2020 .\end{array}$ & $\begin{array}{l}\text { Dal'bosco et al. } \\
\qquad(2020)\end{array}$ & 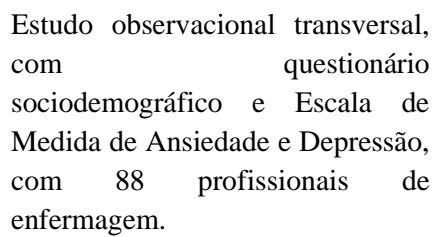 & $\begin{array}{l}\text { Identificar a prevalência e fatores associados } \\
\text { à ansiedade e depressão em profissionais de } \\
\text { enfermagem que atuam no enfrentamento da } \\
\text { COVID-19 em hospital universitário. }\end{array}$ \\
\hline $\begin{array}{l}\text { A enfermagem frente ao } \\
\text { processo de morte e morrer: uma } \\
\text { reflexão em tempos de } \\
\text { Coronavírus. }\end{array}$ & $\begin{array}{l}\text { De Paula et al. } \\
\qquad(2020)\end{array}$ & $\begin{array}{l}\text { Estudo de análise reflexiva } \\
\text { baseada na leitura, análise e } \\
\text { interpretação de artigos e relatórios } \\
\text { do Ministério da Saúde e do } \\
\text { Conselho Federal de Enfermagem. }\end{array}$ & $\begin{array}{l}\text { Refletir sobre os desafios da enfermagem } \\
\text { diante do processo de morte e morrer em face } \\
\text { a pandemia por Coronavírus. }\end{array}$ \\
\hline $\begin{array}{l}\text { Impactos na Saúde Mental e } \\
\text { Intervenções Psicológicas Diante } \\
\text { da Pandemia do Novo } \\
\text { Coronavírus (COVID-19). }\end{array}$ & $\begin{array}{l}\text { Schmidt et al. } \\
\qquad(2020)\end{array}$ & $\begin{array}{l}\text { Revisão de literatura técnico- } \\
\text { científica produzida em diferentes } \\
\text { países, na perspectiva de sumarizar } \\
\text { desenvolvimentos recentes ligados } \\
\text { à COVID-19. }\end{array}$ & $\begin{array}{l}\text { Sistematizar conhecimentos sobre os } \\
\text { impactos na saúde mental e intervênções } \\
\text { psicológicas diante da pandemia do novo } \\
\text { coronavírus. }\end{array}$ \\
\hline
\end{tabular}

Fonte: Autores.

Foram encontradas similaridades de temáticas abordadas nos estudos e, assim, desenvolveram-se quatro categorias temáticas: "Cenário da pandemia no Brasil e no mundo"; "Perfil profissional dos trabalhadores em saúde acometidos por danos a saúde durante a Pandemia do COVID-19"; "Principais danos à saúde que acometeram os profissionais de saúde durante a Pandemia do COVID-19"; "Estratégias elencadas para restabelecimento da condição de saúde dos profissionais de saúde atuante na linha de frente contra a Pandemia do COVID-19".

\section{Discussão}

\section{Cenário da pandemia no Brasil e no mundo}

A pandemia da COVID-19 apresentou-se como o maior desafio sanitário em escala global deste século, infectando diversos países. Poucos meses depois do início da pandemia na China, já ocorriam mais de 2 milhões de casos e 120 mil mortes no mundo pelo novo coronavírus. Os Estados Unidos ocuparam a primeira posição mundial ao serem detectados 2.220.961 casos do vírus (Wernek \& Carvalho, 2020). No Brasil, a doença se propagou rapidamente e em menos de um mês após a confirmação do primeiro caso, já havia transmissão comunitária em algumas cidades, cuja primeira notificação ocorreu em 20 de março de 2020, 3 dias após o primeiro óbito por COVID-19- (Oliveira et al., 2020).

Ainda em março de 2020, o Governo do Estado de São Paulo, epicentro da pandemia no país, promulgou o Decreto $\mathrm{n}^{\circ}$ 64.881, no qual se definiram medidas de isolamento social e quarentena estadual (Diário Oficial da União, 2020). Sucessivamente, Rio de Janeiro, Distrito Federal e Minas Gerais também decretaram ações de isolamento ou distanciamento social, de forma a conterem a curva de transmissão do COVID-19. Nesse período foram detectado 49.976 óbitos no país (Brasil, 2020). 
De acordo com o Conselho Federal de Enfermagem (COFEN, 2021), foram registados até 04 de maio de 2021, mais de 776 mortes de enfermagem decorrentes da infecção pela Covid-19, no Brasil. A região Sudeste foi a que apresentou maior número de mortes desses profissionais desde o início da pandemia. Foram 219 óbitos, o que corresponde a 28\% do total de óbitos desses profissionais no país. Em relação aos estados, São Paulo, Amazonas e Rio de Janeiro tiveram o maior número de mortes no Brasil, com 101, 80 e 65 casos, respetivamente.

\section{Perfil profissional dos trabalhadores em saúde acometidos por danos à saúde durante a pandemia do Covid-19}

Em todo o mundo, milhões de profissionais de saúde estão na linha de frente do cuidado dos pacientes com COVID19 que requerem atendimento ambulatorial ou hospitalar, sendo o maior contingente composto por profissionais de enfermagem. De acordo com Lima et al (2020), um cálculo feito pela American Nurses Association sinaliza que, aproximadamente, 3,8 milhões de enfermeiros nos Estados Unidos; e mais de 20 milhões de enfermeiros em todo o mundo estão envolvidos no enfrentamento da pandemia.

Estima-se que na China, 22 dos cerca de 3.300 profissionais de saúde infectados evoluíram a óbito (Adams \& Walls, 2020). Na Itália, 4.824 profissionais de saúde foram infectados por COVID-19. Dentre eles, foram registrados 24 óbitos na classe médica, o que levou a Federação Italiana de Profissionais de Saúde a considerar que o modelo de cuidados aos trabalhadores de saúde desse estabelecimento de saúde mostrou-se inadequado ao lidar com o surto do novo Coronavírus (Anelli, 2020).

No Brasil, desde o início da pandemia 226 profissionais de saúde morreram e outros 257 mil foram infectados pelo novo vírus;. Dentre as mortes em decorrência da COVID- 19, as categorias com maior taxa de mortalidade foram técnicos e auxiliares de enfermagem $(38,5 \%)$, seguidos por médicos $(21,7 \%)$ e enfermeiros $(15,9 \%)$. Já as taxas de morbidade foram técnicas e auxiliares de enfermagem $(34,4 \%)$, seguidos de enfermeiros $(14,5 \%)$, médicos $(10,7 \%)$ e agentes comunitários de saúde (4,9\%) (Brasil, 2020).

Estudo realizado por Duarte et al. (2020), com 184 dos profissionais de saúde hospitalizados por COVID-19 no Brasil, identificou predominância do sexo feminino, na faixa etária entre 40 - 50 anos e raça/cor branca. Os profissionais com maior número de acometimentos foram da categoria da enfermagem e da medicina. Metade dos adoecidos apresentavam comorbidades como cardiopatia, hipertensão arterial sistêmica, diabetes melitus e asma. Dos 27 que evoluiram para óbito, $89 \%$ apresentavam comorbidades, $44 \%$ eram atuantes na área de enfermagem e 33\% na medicina, com predomínio do sexo masculino, raça/cor branca, idade média de 44 anos.

Já um estudo que observou a saúde mental dos profissionais de enfermagem durante o período da pandemia, realizado no Hospital Regional dos Campos Gerais da Universidade Estadual de Ponta Grossa, obteve como resultado a prevalência de ansiedade e depressão, em maior destaque em profissionais do sexo feminino, com mais de 40 anos, casadas ou em união estável, cor/raça branca, escolaridade de ensino médio superior ou pós-graduação, concursadas, com renda superior a $\mathrm{R} \$ 3.000,00$, com regime de trabalho de 40 horas semais e tempo de atuação no hospital de 1 a 5 anos (Dal'Bosco et al., 2020).

\section{Principais danos à saúde que acometeram os profissonais de saúde durante a pandemia do Covid-19}

Segundo Teixeira et al. (2020), o principal problema de saúde que afetou os profissionais envolvidos diretamente no cuidado aos pacientes sintomáticos ou diagnosticados com a infecção provocada pelo COVID-19 foi o medo pelo risco de contaminação pela doença, haja vista os; milhares de trabalhadores fa fáderam afastados de suas atividades laborais por terem se infectado. Cabe destacar a notoriedade, durante o período da pandemia, episódios de medo, ansiedade e depressão persistentes entre os profissionais atuantes na linha de frente, justificando-se a presença desses sentimentos pela exposição aos 
fatores estressantes, como a convivências com altas taxas de mortalidade, o racionamento de equipamentos de proteção individual, a falta de acesso a respiradores e a outros suprimentos essenciais à saúde (Ladeia et al., 2020).

Os profissionais de saúde que enfrentam a crise causada pela pandemia do novo coronavírus, sofrem variações quanto aos esforços para harmonizar sua necessidade pessoal e o seu seguimento de trabalho, muitos profissionais não alcançam tal conciliação entre os dois meios por receio e tensão da sociedade, sendo capazes de potencializar episódios estressantes presentes no cotidiano das profissões. (Oliveira et al., 2021).

Segundo Kang et al. (2020), a vivência em um ambiente de enorme pressão, alto risco de infecção, e proteção inadequada contra contaminação, excesso de trabalho, frustração, discriminação, isolamento, assistência a pacientes com emoções negativas, falta de contato com a família e exaustão, causaram nos profissionais problemas relacionados à saúde mental, que não apenas afetam a atenção, o entendimento e a capacidade de tomada de decisões dos servidores, mas também podem ter um efeito duradouro em seu bem-estar geral.

Foram evidenciadas lesões cutâneas nos profissionais, devido ao uso de EPIs por tempos prolongados e contínuos na jornada de trabalho, as principais lesões ocasionadas por esses equipamentos foram ressecamento da pele e dermatites, pela intensa higienização das mãos e uso de luvas, e as lesões por pressão (LPP) relacionadas ao uso de máscaras e protetores faciais. A literatura recente relata que os principais locais afetados são osso nasal, bochechas, testa, orelhas e mãos, em virtude do uso de máscaras, face shield, óculos e luvas, causando LPP e eczema (Dutra \& Xavier, 2020).

Para Carvalho et al. (2020), o uso inadequado e rotineiro de EPIs implica forças mecânicas e químicas sobre a pele, agredindo-a e alterando suas características protetoras. Fatores físicos, como pressão sustentada, forças de tensão, fricção e atrito, bem como a umidade e a temperatura, estão diretamente associadas ao desenvolvimento de úlceras e lesões por fricção, principalmente nas regiões de ponta nasal, mãos, bochecha e testa. Estas lesões podem aumentar o risco de infecção, causar dor e cicatrizes, resultando em prejuizo na qualidade de vida. Outrossim, a higienização constante das mãos, acompanhadas do uso de luvas e álcool em excesso, aumentam o risco de dermatites e dermaftoses, podendo causar eritema, ressecamento, descamação, físsuras, prurido, infecções secundárias e agravamento de doenças de pele (Dutra \& Xavier, 2020).

$\mathrm{O}$ medo de se infectar, de morrer e de transmitir tem sido um sentimento presente na vida de muitos profissionais de saúde atuantes na linha de frente contra a pandemia da COVID-19. A morte de pacientes contaminados tem sido bastante desafiadora a ser enfrentada pelos profissionais de saúde, principalmente para a categoria de enfermagem, que acompanham todo o processo de morte e morrer. Diante da situação de morte iminente, os profissionais, em sua maioria, apresentam o sentimento de culpa pelo ocorrido, por não saberem posicionar-se frente ao sofrimento e à dor da perda. Uma consequência desse posicionamento defensivo é a identificação da Síndrome de Burnout nesses, devido ao desgaste e ao sofrimento, suscitada pela exposição crônica aos estressores psicossociais presentes no desempenho das suas atividades laborais (De Paula et al., 2020).

Toescher et al. (2020) identificaram as principais implicações da pandemia na saúde mental da categoria de enfermagem, abordando como principais fatores: a alta demanda de atendimento, exposição prolongada em ambientes críticos, risco contínuo de infecção, uso prolongado e escassez de EPIs, gerência do sofrimento de pacientes e familiares, falta de contato com a familia, conflitos interpessoais, pensamentos recorrentes sobre a pandemia e relacionados à morte e ao morrer, estigma por trabalhar com pacientes infectados e desmitificação de notícias falsas constantemente.

Estratégias elencadas para restabelecimento da condição de saúde dos profissionais de saúde atuantes na linha de frente contra a pandemia do Covid-19

Carvalho et al. (2020) recomendam medidas preventivas para evitar as complicações dos EPIs relacionados à pele, tais como cuidados antes e após a utilização dos EPIs, utilizando cremes hidratantes, protetores de barreira; utilização de 
material de interface, como bandagem ou esparadrapo entre o EPI e a pele nas zonas de adesão/pressão/fricção; realizar a lavagem das mãos associado ao uso de substâncias cosméticas que podem ajudar no processo de retenção e redução da perda de água transepidérmica; programar minutos de alívio de pressão dos EPIs, planejando turnos de rotação mais curtos em equipamentos de alta intensidade protetora, sempre tomando cuidado com o risco de contaminação; e tratar, proteger e evitar o uso de máscara e óculos de proteção sobre áreas afetadas.

Quanto à atenção psicossocial, em 26 de março de 2020, no Brasil, foi publicada a Resolução CFP nº 4/2020, que disponibiliza a prestação de serviços psicológicos por meios de tecnologia a informação e da comunicação após realização do "Cadastro e-PSI". Esta Resolução mostrou-se importante para o atendimento a pessoas e grupos em situações de urgência, emergência e desastres, violação de direitos ou violência, com o objetivo de minimiar as implicações psicológicas diante da Pandemia (Schmidt et al., 2020).

Além disso, manter a saúde mental é essencial durante o período de pandemia. Portanto, deve-se procurar se alimentar e se hidratar de maneira adequada, praticar atividades físicas, manter o contato social, mesmo à distância, praticar ou exercitar a fé, caso tenha, realizar atividades prazerosas, manter o sono reparador e fazer pausas do expediente, se necessário (Ramalho, 2020).

A categoria de enfermagem é abordada como uma das que mais tem apresentado sofrimento mental. Com isso, Toescher et al. (2020) corroboram com as estratégias para o enfrentamento das implicações da pandemia na saúde mental anteriormente citadas, como atentar às necessidades básicas: alimentação, hidratação sono regular, evitar hábitos prejudiciais, criar uma rotina de trabalho e autocuidado, permanecer concentado a uma rede sócio afetiva, manter-se atualizado em fontes de informação confiáveis, praticar resiliência, manter a fé e atividades religiosas e/ou espirituais e buscar ajuda de um profissional de saúde quando necessário para estabilização emocional.

No Brasil, o cuidado em saúde mental dos profissionais de saúde ainda está sendo estruturado através das Secretarias Municipais e Estaduais da Saúde com apoio das universidades públicas e centros de pesquisa, que tem fornecido subsídios teóricos com base em evidências científicas produzidas em outros países. Nessa perspectiva, vem sendo propostos planos de contingência para atenção psicossocial e promoção da saúde mental dos trabalhadores da saúde em vários estados , com ações desenvolvidas para o acolhimento e atendimento à crise, por meio de intervenção psicossocial rápida, mas também pela garantia de um conjunto de ações de caráter preventivo, no sentido de minimizar as probabilidades dos profissionais sofrerem danos psicossociais a médio prazo e especialmente ações que promovam ambientes protegidos e favoráveis à saúde mental dos trabalhadores da saúde (Fiocruz, 2020).

\section{Considerações Finais}

Aflições causadas aos profissionais de saúde frente à pandemia da COVID-19 é um assunto que precisa de maior abordagem. Por ser um tema recente, houve limitação na busca dos impactos causados a estes profissionais. Acredita-se que a implantação de medidas de redução de danos causados aos profissionais de saúde e a prevenção da contaminação recomendada pela OMS colaborem na reduação destas complicações. Devendo ocorrer de forma criteriosa, independentemente do nível de atenção à saude ou do desenvolvimento econônico do país, mesmo sabendo que países desenvolvidos também tiveram dificuldades em adaptar-se a este novo cenário assistencial.

Frente a isso, é observado no estudo que a categoria que mais sofreu com os danos decorrentes da pandemia foi a de enfermagem, devido a sobrecarga de trabalho na luta contra o novo Coronavírus, o que explicita a necessidade de debater formação, funções, condições de vida, condições de trabalho e rumos. Necessita-se, ainda, repensar o sistema de saúde e as formas para o enfrentamento de surtos, epidemias e pandemias. Somado a isto, ressalta-se que as construções práticas da dimensão assistencial possuem grande repercussão no momento atual de consolidação da autonomia profissional. 
Vive-se um momento no qual a Enfermagem se apresenta como força expressiva e necessária no combate ao novo Coronavírus. Não obstante, o despreparo diante dos processos de morte e morrer causados pelas lacunas abertas nos cursos de formação ou mesmo pela insistente reprodução de modelos de cuidados centrados na dinâmica do paradigma biomédico, tecnicista, reducionista, acarreta nos profissionais sofrimentos psicológicos e físicos no enfrentamento da terminalidade.

\section{Referências}

Adams, J. G., \& Walls. R. M. (2020). Supporting the Health Care Workforce During the COVID-19 Global Epidemic. JAMA; 323(15):1439-1440.

Araujo, S. E. A. Leal, A., Centrone, A. F. Y., Teich, V. D., Malheiro, D. T., Cypriano, A. S., Neto, M. C. \& Klajner, S. (2021). Impacto da Covid-19 sobre o atendimento de pacientes oncológicos: experiência de um centro oncológico localizado em um epicentro Latino-Americano da pandemia. Eisthen. São Paulo.

Avanian, J. Z. (2020). Mental Health Needs of Health Care Workers Providing Frontline COVID-19 Care: Editor's Comment COVID-1. JAMA.

Aydogdu, A. L. F. (2020). Violência e discriminação contra profissionais de saúde em tempos de novo coronavírus. J. nurs. health.

Brasil, Ministério da Saúde. (2020) Boletim Epidemiologico SUS. vol 5, Saúde mental, https://portalarquivos2.saude.gov.br/images/pdf/2 020/janeiro/28/Boletim- epidemiologico-SVS-28jan20.pdf.

Brasil, Ministério da Saúde. (2020). Boletim Epidemiologico SUS. vol 5, Saúde mental, https://portalarquivos2.saude.gov.br/images/pdf/ 2020/janeiro/28/Boletim- epidemiologico-SVS-28jan20.pdf.

Brasil, Ministério da Saúde. (2020). Caderno Humaniza SUS. vol 5, Saúde mental, .

Brasil. Ministério da Saúde. (2021). Painel Coronavírus: atualizado em: 19/04/2021 19:20. https://covid.saude.gov.br/.

Carvalho, R. S., Augusto ,G. R. \& Schoen, I. P. (2020). Utilização de equipamentos de proteção individual em época de COVID-19. Glob Acad Nurs. 1(1):e6.

Conselho Federal De Enfermagem - COFEN. (2021). Mortes entre profissionais de Enfermagem por Covid-19 cai $71 \%$ em abril. http://www.cofen.gov.br/mortes-entre-profissionais-de-enfermagem-por-covid-19-cai-71-em-abril_86775.html.

Dal' Bosco, E. B, Floriano, L. S. M., Skupien, S. V., Arcaro, G., Martins., A. R., \& Anselmo, A. C. C. (2020). A saúde mental da enfermagem no enfrentamento da COVID-19 em um hospital universitário regional. Revista Brasileira de Enfermagem.

De Paula, G. S., Gomes, A. M. T., Franla, L. C. M., Neto, F. R.A. \& Barbosa, D. J. (2020). A enfermagem frente ao processo de morte e morrer: uma reflexão em tempos de Coronavírus. https://docs.bvsalud.org/biblioref/2020/07/1104066/13-a-enfermagem-frente-ao-processo-de-morte-e-morrer-umarefle_eaHsaZB.pdf.

Duarte, M. M. S., Haslett, M. I. C., Freitas, L. J. A., Gomes, N. T. N., Silva, D. C. C., Percio, J., Wada, M. Y., Fantiato, F. F. S. T., Almeida, W. A. F., Silva, D. A., Gava, C., França, G. V. A., Macário, E. M., Baêta, K. F., Malta, J. M. A. S. \& Alves A. J. S. Descrição dos casos hospitalizados pela COVID-19 em profissionais de saúde nas primeiras nove semanas, Brasil, 2020. Epidemiol. Serv. Saúde 29

Dutra, J. I.S. \& Xavier, M. A. (2020). Lesões De Pele Relacionada Ao Uso De Dispositivos Édicos No Enfrentamentoao Covid-10. Medidas De Prevenção E Tratamento. Universidade Federal Do Rio Grande Do Sul, Santa Cruz. https://facisa.ufrn.br/noticia/1282/cartilha-sobre-lesoes-de-pele- relacionadas-ao-usode-dispositivos-medicos-no-enfrentamento-ao-covid-19-medidas- de-prevencao-e-tratamento.

Fundação Oswaldo Cruz (FIOCRUZ). (2020). Saúde mental e atenção psicossocial na pandemcia COVID-10. Recomendações para gestores. https://www.fiocruzbrasilia.fiocruz.br/wp-content/uploads/2020/04/Sa\%c3\%bade-Mental-e-Aten\%c3\%a7\%c3\%a3o-Psicossocial-na-Pandemia-Covid-19recomenda\%c3\%a7\%c3\%b5es-para-gestores.pdf.

Helioterio, M. C., Lopes, F. Q. R. S., Sous, C. C., Souza, F. O., Pinho, P. S., Sousa, F. N. F., Araújo, T. M. (2020). Covid-19: Por que a proteção de trabalhadores e trabalhadoras da saúde é prioritária no combate à pandemia? Trab. Educ e Saúde.

Kang, L., Li, Y. \& Hu, S. (2020). The Mental Health Of Medical Workers In Wuhan, China Dealing With The 2019 Novel Coronavírus. Lancet Psychiatry 7(3): 14 .

Koh, David (comp.) (2020). Occupational risks for COVID-19 infection. 2020. US National Library of Medicine National Institutes of Health Search database. https://www.ncbi.nlm.nih.gov/pmc/articles/PMC7107962/.

Ladeia, D. N., Silva, A. F., Gonçalves, B. B. S., Damasceno, C. M. C., Vieira, J. P. G., Silva, J. A.L., Lopes, L. M. M., Queiroz, N. O., Lopes, A. G. (2020). Análise da saúde mental na população geral durante a Pandemia do Covid-10. https://www.scielo.br/scielo.php/saude/article/view/3925.

Lima et al. (2020). A Saúde Dos Trabalhadores De Saúde No Enfrentamento Da Pandemia Da Covid-19. Boletim Covida Pandemia De Covid-19. Edição: 05. http://www.saude.ba.gov.br/wp-content/uploads/2020/05/Boletim-CoVida-5_Edit_.pdf-1.pdf.

Luz, A., Noronha, R., Navarro, T. (2020). COVID - 19: medidas de prevenção de lesão por pressão ocasionadas por equipamentos de proteção individual em profissionais da saúde. Revista Enfermagem Atual In Derme, 93, e-020011.

Oliveira, E. N., Costa, M. S. A., Nascimento, P. I. da F. V. do., Rodrigues, C. S., Andrade, C. S. G. de., Mendonça, J. M. F., Pinto, M. R., França, S. da S. \& Lima, G. F. With the word, health professionals who are at the front line of COVID-19 combat. Research, Society and Development, 9 , e30985145, 2020. 10.33448/rsd-v9i8.5145. https://rsdjournal.org/index.php/rsd/article/view/5145. 
Research, Society and Development, v. 10, n. 8, e17610817169, 2021

(CC BY 4.0) | ISSN 2525-3409 | DOI: http://dx.doi.org/10.33448/rsd-v10i8.17169

Oliveira, W., Duarte, E., França, G.V. A. \& Garcia, L. P. (2020). Como o Brasil pode deter a Covid-19. https://www.scielo.br/pdf/ress/v29n2/2237-9622-ress29-02-e2020044.pdf.

Ramalho, A. O. (2020). Lesões De Pele Relacionadas Ao De Equipamentos De Proteção De Individual Em Profissionais. Estratégias De Prevenção Frente À Pandemia Por Covid-19. Associação Brasileira De Estomatoterapia - Sobest. http://www.abennacional.org.br/site/wpcontent/uploads/2020/04/LPRDM_COVID19_Manual-Versa\%CC\%83o-Portugues.pdf.

Schmidt, B., Crepaldi, M. A., Bolze, S. D. A., Neiva-Silva, L. \& Demench, L. M. (2020). Impactos na Saúde Mental e Intervenç̃̃es Psicológicas Diante da Pandemia do Novo Coronavírus (COVID-19). https://preprints.scielo.org/index.php/scielo/preprint/view/58/69.

Teixeira, C. F., Soares, C. M. \& Souza, E. A. (2020). A Saúde Dos Profissionais De Saúde No Enfrentamento Da Pandemia Do Covid-19. Fundação Oswaldo Cruz. https://renastonline.ensp.fiocruz.br/sites/default/files/arquivos/recursos/a-saude-dos- profissionais-de-saude-no-enfrentamento-da-pandemia-de-covid19.pdf.

Toescher, A. M. R, Barlem, J. G. T, Balem, E. L. D., Castanheira, J. S. \& Toescher, R. L. (2020). Saúde mental de profissionais de enfermagem durante a pandemia de COVID-19: recursos de apoio. Esc. Anna Nery. https://www.scielo.br/scielo.php?script=sci_arttext\&pid=S1414-81452020000500503\&tlng=pt.

Yan, Y; Chen, H, Chen L, Cheng, B. (2020). Consensus of Chinese experts on protection of skin and mucous membrane barrier for healthcare workers fighting against coronavirus disease 2019. Dermatol Ther; e13310.

Yu. J. et al. (2020). SARS-COV-2 Transmission in patients with câncer at a tertuary cary hospital in Wuhan. JAMA oncology, Wuhan, 6, 1108-110.

Werneck, G. L., Carvalho, M. S. (2020). A pandemia de COVID-19 no Brasil: crônica de uma crise sanitária anunciada. Cad. Saúde Pública, 36, e00068820. http://cadernos.ensp.fiocruz.br/csp/artigo/1036/a-pandemia-de-covid-19-no-brasil- cronica-de-uma-crise-sanitaria-anunciada.

World Health Organization (WHO). (2021). Coronavirus (COVID-19) Dashboard. https://covid19.who.int/.

World Health Organization. (2020). Rational use of personal protective equipment for coronavirus disease 2019(COVID-19): Interim guidance. Genebra: WHO. https://apps.who.int/iris/bitstream/handle/10665/331215/WHO-2019-nCov- IPCPPE_use-2020.1-eng.pdf. 